This item was submitted to Loughborough's Research Repository by the author.

Items in Figshare are protected by copyright, with all rights reserved, unless otherwise indicated.

\title{
Fabrication of artificial dielectrics via stereolithography based 3D-printing
}

PLEASE CITE THE PUBLISHED VERSION

https://doi.org/10.23919/EuCAP48036.2020.9135734

PUBLISHER

IEEE

VERSION

AM (Accepted Manuscript)

\section{PUBLISHER STATEMENT}

(c) 2020 IEEE. Personal use of this material is permitted. Permission from IEEE must be obtained for all other uses, in any current or future media, including reprinting/republishing this material for advertising or promotional purposes, creating new collective works, for resale or redistribution to servers or lists, or reuse of any copyrighted component of this work in other works.

\section{LICENCE}

All Rights Reserved

\section{REPOSITORY RECORD}

McGhee, Jack, Tom Whittaker, Jacob Moriarty, Jamie Northedge, Shiyu Zhang, Darren Cadman, William Whittow, and J. C. Vardaxoglou. 2020. "Fabrication of Artificial Dielectrics via Stereolithography Based 3dprinting". Loughborough University. https://hdl.handle.net/2134/11890122.v1. 


\title{
Fabrication of Artificial Dielectrics via Stereolithography Based 3D-Printing
}

\author{
Jack R. McGhee ${ }^{1 *}$, Tom Whittaker ${ }^{1}$, Jacob Moriarty ${ }^{1}$, Jamie Northedge ${ }^{1}$, Shiyu Zhang $^{1}$, Darren Cadman ${ }^{1}$, Will \\ Whittow ${ }^{1}$, John (Yiannis) Vardaxoglou ${ }^{1}$
}

${ }^{1}$ Wolfson School of Mechanical, Electrical and Manufacturing Engineering, Loughborough University, Loughborough, United Kingdom, J.McGhee@lboro.ac.uk*

\begin{abstract}
In this research, stereolithography (SLA) based additive manufacturing (AM) has been investigated as a fabrication method for producing artificial dielectrics. Initially, the effect of the curing time on the microwave electromagnetic properties (X-band) on the photoinitiated resin used was measured and found to be negligible after 15 minutes of $U V$ curing. Artificial dielectric isotropic and anisotropic lattice structures were then designed and fabricated, allowing for varying permittivity between 1.23 and 2.80 through the control of the structure's density. As a demonstration of the ability to grade permittivity through a high-resolution printing process, lattice structures were embedded into solid substrates. The ability to do this allowed for the printing of a graded permittivity substrate which is showcased in a design for a circularly polarized patch antenna.
\end{abstract}

Index Terms - artificial dielectrics, additive manufacturing, graded index materials, circular polarization.

\section{INTRODUCTION}

In the field of electromagnetics (EM), most applications are fabricated through traditional manufacturing methods such as injection molding and metal plating, milling and micro-machining. However, as the field of electromagnetic meta-materials grows, complex, three-dimensional (3D) are required to realize application designs which provide new functionalities and improved performance for filters, lenses and antennas [1-3]. There is also an increased drive for bespoke substrates with electromagnetic properties that can be tailored, enabling engineers to rapidly design and optimization structures for antennas, lenses and filters. The best candidate to achieve the manufacturing of these structures is additive manufacturing (AM).

Additive manufacturing is a manufacturing technique more commonly known as $3 \mathrm{D}$ printing is a method of manufacturing and rapid prototyping defined as 'a process of joining materials to make objects from $3 \mathrm{D}$ model data, usually layer upon layer, as opposed to subtractive manufacturing methodologies' [4]. The potential for AM to decentralize manufacturing was once hailed as the 'next industrial revolution' [5]. It allows for the fabrication of complex geometries that can save on waste material, weight and design costs, offering rapid customizability of functional materials. There are a variety of additive manufacturing methods with the most commonly known technique being fused filament fabrication, were polymers or polymer / metal or polymer / ceramic composites are melted and extruded through a nozzle. Fused filament fabrication has many advantages; however, a major disadvantage is print resolution and reproducibility with current equipment. Printing resolution can be increased by using other AM methods. Stereolithography (SLA), this is an additive manufacturing technique which fabricates 3D structures by the photoinitiated curing of a resin. The photoinitiation is typically performed via laser, allowing for the relatively high-resolution additive manufacture of polymer and ceramic structures compared to other manufacturing techniques [6].

Due to this, SLA provides a lot of design freedom for engineering and is an excellent candidate for fabricating bespoke substrates and metamaterial periodic structures for electromagnetic applications. Periodic structures printed via SLA have been exploited in mechanical metamaterials but are yet to be widely used for EM based applications [7-8]. Some fundamental EM based 'meta-material' applications are artificially engineered substrates. These substrates can have permittivity tailored to the needs of the engineer through in the inclusion of air voids or metals [9-10]. There is no literature investigating the effects of air voids on SLA printed materials, although the resolution is so much greater allowing for a higher degree of tailoring. This higher resolution also allows for thin layers of polymer to be printed to encapsulate the lattice, allowing very low permittivity substrates to be metalized. Through this method, the SLA printed lattices are incorporated into the substrate of a patch antenna to achieve circular polarization. In this research, computer-aided-design (CAD) lattice generation tools are then used to model three-dimensional (3D) lattice structures to enable the fabrication of air void based artificial dielectric substrates through SLA 3D-printing. In this paper we investigate the X-band EM properties of polymer resin for SLA based additive manufacturing with respect to UV curing time. 


\section{EFFECT OF UV CURING ON SLA RESIN}

This research was performed using a Formlabs 2 SLA Printer with clear resin (Formlabs, RS-F2-GPCL-04). All samples in this research were printed with a $50 \mu \mathrm{m}$ layer height and after printing were immediately washed in isopropyl alcohol (Alfa Aesar, 99\%) for 10 minutes to clean off any excess resin. Although the resin is cured via laser (405nm) during the printing process, it can be cured further with UV light after printing. Characterization of the bulk material was initially performed with respect to curing times to determine whether the UV curing time influenced the Xband microwave dielectric properties of the clear resin, namely the permittivity and loss tangent. Samples were cured using a Form Cure UV curing system, with the temperature kept constant at the manufacturers recommended setting of $60^{\circ} \mathrm{C}$. Curing times were varied between 30 seconds and 21.5 hours and compared to references samples which did not undergo the additional curing step. All samples were designed and printed to be measured by a rectangular WR90 waveguide $(10.16 \mathrm{x}$ $22.86 \mathrm{~mm})$. The electromagnetic properties between 8$12 \mathrm{GHz}$ were measured using a Vector Network Analyzer (Anritsu MS46522B VNA) and the electromagnetic properties extracted using the Nicolson-Ross Weir method $[11,12]$. Samples for measuring the UV curing effects were fabricated to be $100 \%$ at $2 \mathrm{~mm}$ thick. Data for the effects of UV curing on the mean X-band permittivity $\left(\varepsilon_{\mathrm{r}}\right)$ and loss tangent $(\tan \delta)$ including standard error of the mean can be seen in Figure 1. Each data point represents the mean of five samples.

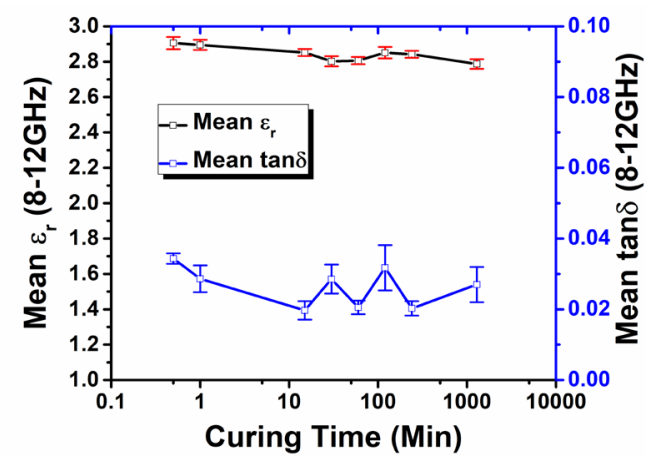

Fig. 1. Curing Time of 3D-Printed SLA Samples versus X-band permitivitiy and loss tangent

Uncured samples had an initial mean $\varepsilon_{\mathrm{r}}$ of 2.823 and mean $\tan \delta$ of 0.04 . As can be seen in Figure 1, for the $100 \%$ solid samples there is a slight downward trend in the permittivity with an increasing UV curing time. However, the effect of the curing time on the loss tangent after an initial drop with 30 seconds curing, is negligible with all points falling within the standard error. It can therefore be determined that after 30 seconds of curing, there are no discernable effect on the loss tangent. Due to this, for the fabrication of the lattice based artificial dielectrics, the manufacturer recommended UV curing time of 15 minutes was used for all samples.

\section{LATTICE DESIGN AND FABRICATION}

Autodesk's Netfabb (Premium, 2019) was the computeraided-design (CAD) package used for designing the artificial dielectrics. Lattices were designed to fit into two categories, isotropic and anisotropic. As shown in Figure 2(a-b), an ' $\mathrm{X}$ ' unit cell type was used, with the isotropic unit-cell size kept constant at $2.0 \mathrm{~mm}(\mathrm{x}), 2.0 \mathrm{~mm}(\mathrm{y})$ and $2.0 \mathrm{~mm}(\mathrm{z})$. For anisotropic lattices, the unit cell sizes were also kept constant at $2.0 \mathrm{~mm}(\mathrm{x}), 2.5 \mathrm{~mm}(\mathrm{y})$ and $2.0 \mathrm{~mm}(\mathrm{z})$ with the anisotropy in the same axis as the electric field vector. The volume fraction of the substrate was altered by changing the thickness of the lattice beams between $0.3-0.6 \mathrm{~mm}$. This creates a theoretical volume fraction range of $18-80 \%$.

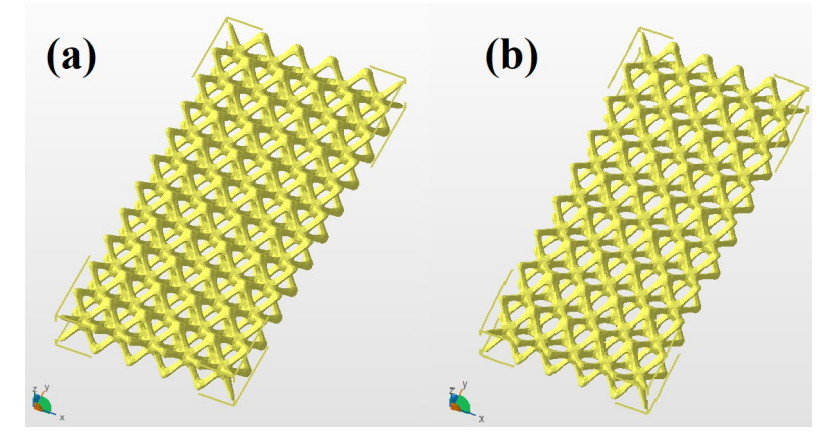

Fig. 2. CAD Designs of Lattice Structures designed to be (a) isotropic and (b) anisotropic

Samples were printed, washed and cured. To ensure the lattice had printed correctly and that the washing procedure adequately removed excess resin, low magnification SEM (Hitachi TM3030Plus Benchtop Scanning Electron Microscope) images were taken of the lattices. Both images of the samples and the scanning electron micrographs can be seen in Figure 3(a-d).

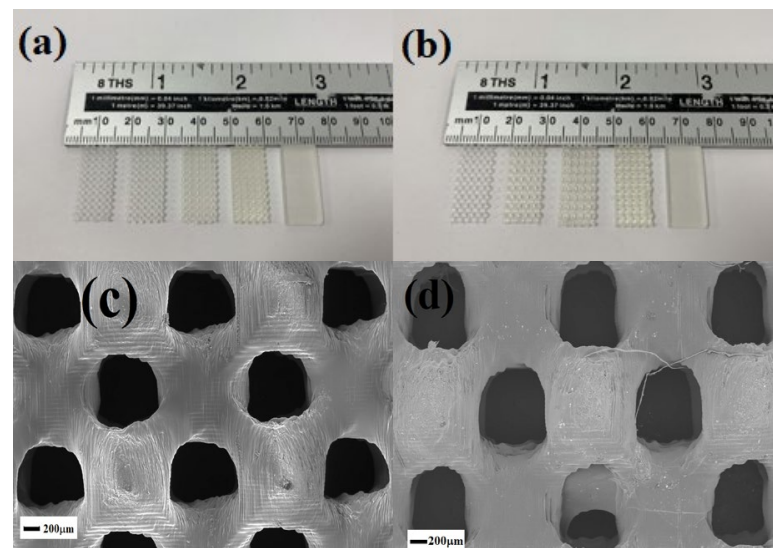

Fig. 3. Overview of printed lattices showing (a) isotropic and (b) anisotropic, (c) SEM of isotropic and (d) SEM of anisotropic unit cells 


\section{ARTIFICIAL DIELECTRIC CHARACTERISATION}

When fabricating additively manufactured artificial dielectrics, the density of the sample is important for determining the volume fraction of air. During the design of the lattice structures, the Autodesk Netfabb software calculates the theoretical volume of the material within the sample design dimensions. Once fabricated, each sample had the dimensions and weight taken in order to calculate the true volume fraction of material versus space. Samples were then, again measured in a rectangular WR90 waveguide and mean permittivity and loss tangents for the X-band were calculated. Firstly, analyzing the four isotropic unit cell designs with data shown in Figure 4(a) with standard error for the permittivity shown in red and the loss shown in blue, the mean permittivity and loss is plotted versus the actual volume fraction of the lattice. By varying the volume fraction of the lattice and therefore altering the fraction of air inside the structure, the relationship of lattice volume to permittivity is linear with an R2 of 0.9972 enabling the predictable customization of the permittivity through the design of the lattice. The observed range of customizable rel. permittivity was between 1.2578 and 2.80228. The mean loss tangent of the samples with varying lattice volumes, displays a similar trend ranging from 0.00654 to 0.02854 .
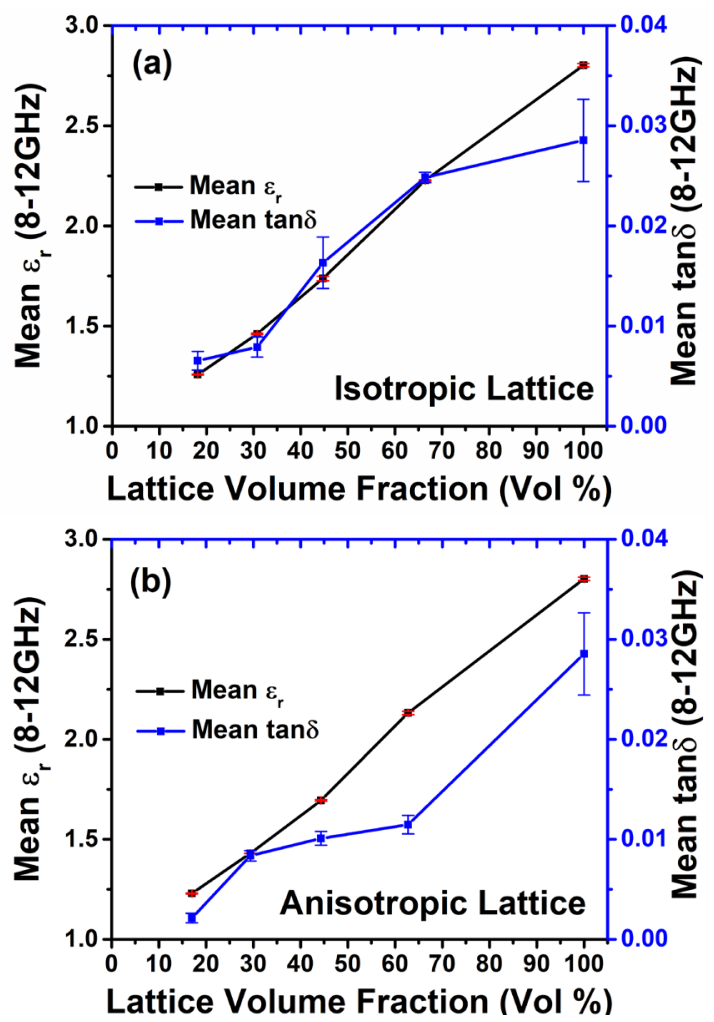

Fig. 4. Lattice volume fraction versus mean X-band permitivity and loss tangent for (a) isotropic and (b) anisotropic additively manufactured lattices

In Figure 4(b), data for the anisotropic lattices can be seen. As mentioned previously, the anisotropy introduced into the unit cell is in the same plane as the E-field. The permittivity versus lattice volume is again linear with an R2 of 0.9974 . The observed range of customizable rel. permittivity was between 1.2298 and 2.80228 . The mean loss tangent of the samples with varying lattice volumes, displays a similar trend ranging from 0.00213 to 0.02854 . Comparing the two datasets, it appears that the anisotropic lattices tend to have generally lower loss than the isotropic lattices whereas the permittivity remains closely linked to the volume fractions of both of polymer and air. Therefore, it appears that by creating anisotropy in the plane of the E-field for a specific application can lower the loss of the SLA polymer resin. However, this would mean the loss would be dependent on the angle of incidence for applications.

\section{ApPlication as a Printed Substrate}

Some electromagnetic applications require graded permittivity structures and substrates with low permittivity, close to that of air. As shown in previous work, normal fused filament fabrication printing of graded permittivity substrates, for low permittivity, open structures with large air pockets are generally required. With these structures to be used as a substrate for EM applications, at least one solid layer is required for metallization (i.e. filter, patch antenna etc.). For the addition of these solid layers, one advantage of the SLA process over FFF is that very thin layers of material between 0.025 to $0.1 \mathrm{~mm}$ can be deposited, lessening the impact on the permittivity. One such application of this is for a circularly polarized $(\mathrm{CP})$ patch antenna which incorporates a heterogenous substrate. The circular polarization is achieved by bringing the resonant frequencies of the TM01 and TM10 modes closer together. The initial dimensions of the patch were calculated at $39.6 \mathrm{~mm} \times 31.2 \mathrm{~mm}$ on a $3 \mathrm{~mm}$ thick $100 \%$ solid resin substrate using the method provided in [13]. To increase the resonant frequency of the first TM10 mode, sections of low permittivity lattices were introduced into the substrate along the short edges of the patch. The low permittivity lattice sections have a designed relative permittivity of 1.23 . The patch length was also increased from $31.2 \mathrm{~mm}$ to $33.6 \mathrm{~mm}$ to lower the resonant frequency of the TM01 mode. The antenna's heterogenous substrate is shown in Figure 5(a) where the lattice sections are clearly visible. These low permittivity sections also have the secondary benefit of reducing the losses incurred in the substrate thus increasing antenna efficiency. The patch was constructed using a piece of copper tape cut to size by hand and the ground plane was comprised of more copper tape. The patch is coaxially fed, the feed point is located $4.8 \mathrm{~mm}$ from the long edge and $12.8 \mathrm{~mm}$ from the short edge. Figure 5(b) shows the final patch with the final dimensions.

The simulated and measured reflection coefficients are shown in Figure 6, the axial ratios are shown in Figure 7 and the antenna radiation patterns are shown in Figure 8. The measured patch antenna achieves the best circular polarization ratio of $0.98 \mathrm{~dB}$ at $3.025 \mathrm{GHz}$, at this frequency the return loss is $10.8 \mathrm{~dB}$. There is a small frequency shift of $35 \mathrm{MHz}$ between the simulated and measurement results which is most likely caused by a slightly oversized patch. 
The simulated and measured antenna directivities at their best axial ratio value are $8.96 \mathrm{dBi}$ and $6.07 \mathrm{dBi}$ respectively and their gain values are $8.13 \mathrm{dBi}$ and $3.77 \mathrm{dBi}$. This gives a calculated antenna efficiency of $58.8 \%$ for the measured antenna. The efficiency can be further increased by improving the impedance matching between the patch and the feed.
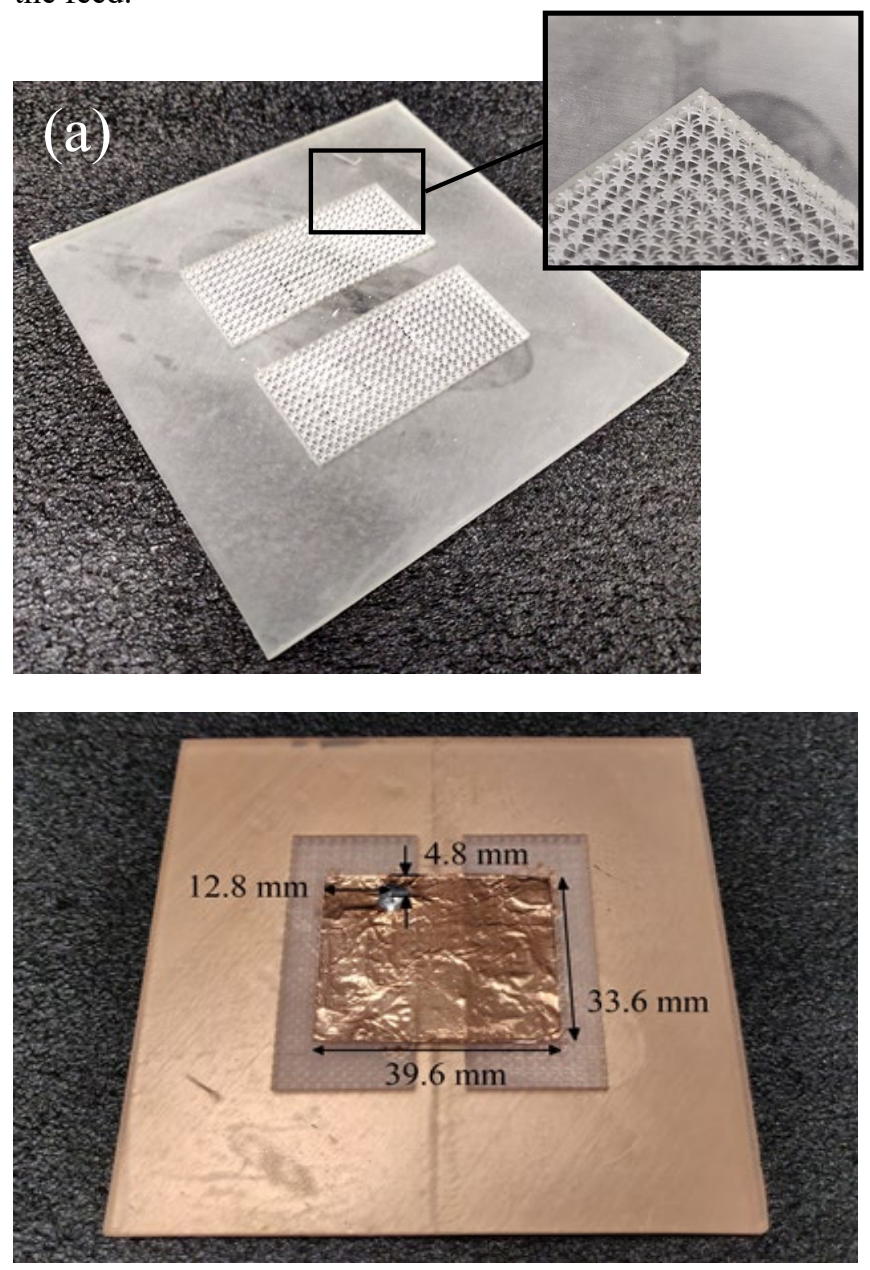

Fig. 5. Photographs of the Circularly polarized patch antenna substrate. (a) heterogeneous substrate with a close-up of the low permittivty latice sections. (b) substrate with the patch added on top bu the broung plane removed.

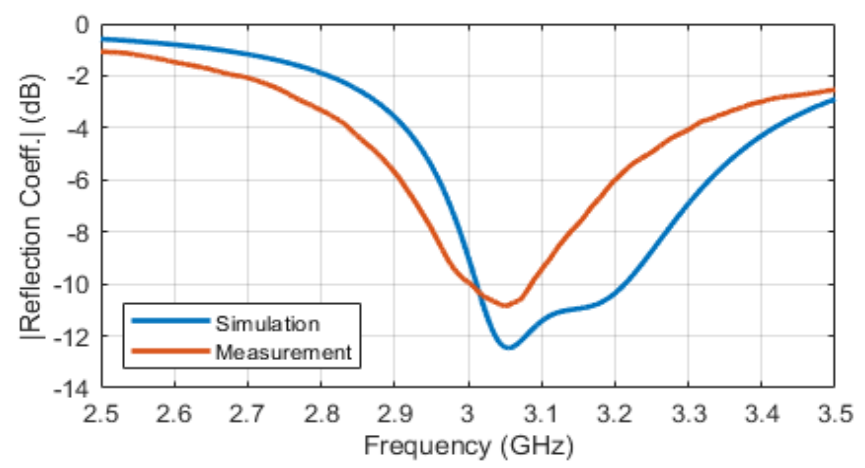

Fig. 6. Simulated and measured reflection coefficents for the circular polarized patch antenna

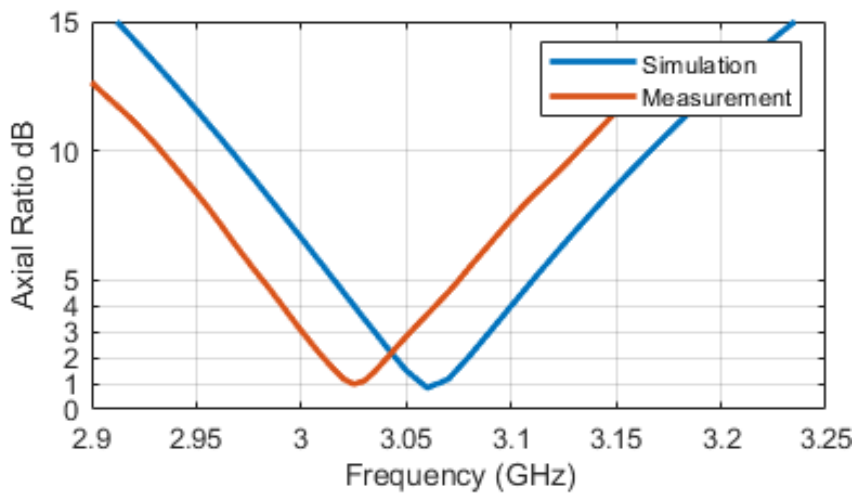

Fig. 7. Simulated and measured axial ration values for the circular polarized patch antenna

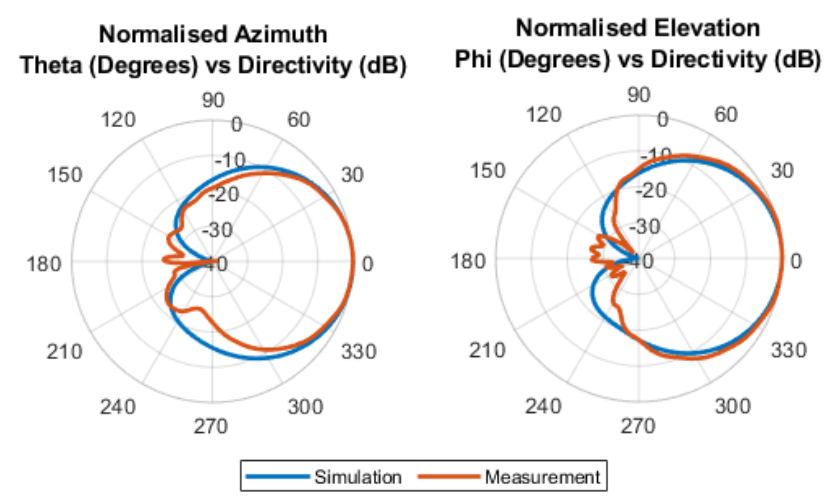

Fig. 8. Normalised measured and simulated left hand polarized radiation patterns for the circular polarized patch antenna

\section{CONCLUSIONS AND FUTURE WORK}

The effects of UV curing on the X-band microwave properties of a common SLA based photocurable resin have been assessed. It has been found that after an initial cure of one minute, no appreciable change in the permittivity or the loss tangent of the photocurable resin was observed. Once the bulk material properties were understood, lattice generation tools were used to design air infill based artificial dielectrics. These were then fabricated and characterized. A linear relationship for permittivity versus lattice volume was found for both isotropic and anisotropic samples, allowing for the customization of the relative permittivity value between 1.23 and 2.80. Lattices were then embedded in a patch antenna substrate to enable CP as a proof of concept, showing that due to the high resolution of the SLA process, low permittivity high resolution lattices can be manufactured. This allows for the design of microwave devices using heterogeneous substrates. The circularly polarized antenna achieved an axial ratio of $0.98 \mathrm{~dB}$ and a directivity of 6.07 with an efficiency of $58.8 \%$

Future work will involve the use of embedded lattices within larger 3D structures and the metallization of said structures for applications such as antennas and metamaterial structures. 


\section{ACKNOWLEDGMENTS}

This work was supported by the EPSRC research grant SYMETA (EP/N010493/1) and was conducted as part of a Nuffield Research Placement, a program organized by the Nuffield Foundation which enables young students to work alongside professional research scientists. The authors acknowledge use of facilities within the Loughborough Materials Characterisation Centre.

\section{REFERENCES}

[1] A. M. Nicolson and G. F. Ross, "Measurement of the Intrinsic Properties of Materials by Time-Domain Techniques," IEEE Trans. Instrum. Meas., vol. 19, no. 4, pp. 377-382, Nov. 1970.

[2] W. B. Weir, "Automatic measurement of complex dielectric constant and permeability at microwave frequencies," Proc. IEEE, vol. 62, no. 1, pp. 33-36, 1974.

[3] M. Kadic, G. W. Milton, M. van Hecke and M. Wegener, "3D metamaterials," Nature Reviews Physics, vol. 1, pp. 198-210, 2019.

[4] A. Vallecchi, D. Cadman, W. G. Whittow, J. C. Vardaxoglou, E. Shamonina and C. J. Stevens, "3-D printed bandpass filters with coupled vertically extruded split ring resonators," IEEE Transactions on Microwave Theory and Techniques, pp. 1 - 12, 2019.

[5] S. Zhang, R. K. Arya, S. Pandey, Y. Vardaxoglou, W. Whittow and R. Mittra, "3D-Printed Planar Graded Index Lens," IET Microwaves, Antennas and Propagation, vol. 10, no. 13, pp. 1411-1419, 2016.

[6] American Society for Testing \& Materials Standard terminology for additive manufacturing technologies. ASTM International, West Conshohocken, PA, 2012.

[7] B. Berman, "3-D printing: The new industrial revolution," Business Horizons, vol. 55, no. 2, pp. 155-162, 2012.

[8] T. D. Ngo, A. Kashani, G. Imbalzano, K. T. Q. Nguyen and D. Hui, "Additive manufacturing (3D printing): A review of materials, methods, applications and challenges," Composites Part B: Engineering, vol. 143, pp. 172-196, 2018.

[9] X. Yu, J. Zhou, H. Liang, z. Jiang and L. Wu, "Mechanical metamaterials associated with stiffness, rigidity and compressibility: A brief review," Progress in Materials Science, vol. 94, pp. 114-173, 2018.

[10] M. Mohsenizadeh, F. Gasbarri, M. Munther, A. Beheshti and K. Davami, "Additively-manufactured lightweight Metamaterials for energy absorption," Materials and Design, vol. 139, pp. 521-530, 2018.

[11] S. Zhang, C. C. Njoku, W. G. Whittow and J. C. Vardaxoglou, "Novel 3D Printed Synthetic Substrates," Microwave and Optical Technology Letters, vol. 57, no. 10, pp. 2344-2346, 2015.

[12] S. Zhang, W. Whittow and J. C. Vardaxoglou, "Additively manufactured artificial materials with metallic meta-atoms," IET Microwaves, Antennas and Propagation, vol. 11, no. 14, pp. 1955 1961, 2017.

[13] C. A. Balanis, Antenna theory. Analysis and design, 3rd ed. Hoboken,: John Wiley \& Sons, Inc., 2005. 